\title{
Spin transfer torque induced paramagnetic resonance
}

\author{
Alexey M. Shakirov,,${ }^{1,2}$, * Alexey N. Rubtsov, ${ }^{1,2}$ and Pedro Ribeiro ${ }^{3,4}$ \\ ${ }^{1}$ Russian Quantum Center, Novaya Street 100A, 143025 Skolkovo, Moscow Region, Russia \\ ${ }^{2}$ Department of Physics, Lomonosov Moscow State University, Leninskie Gory 1, 119992 Moscow, Russia \\ ${ }^{3}$ CeFEMA, Instituto Superior Técnico, Universidade de Lisboa, Av. Rovisco Pais, 1049-001 Lisboa, Portugal \\ ${ }^{4}$ Beijing Computational Science Research Center, 100193 Beijing, China
}

\begin{abstract}
We show how the spin-transfer torque generated by an ac voltage may be used to excite a paramagnetic resonance of an atomic spin deposited on a metallic surface. This mechanism is independent of the environment of the atom and may explain the ubiquity of the paramagnetic resonance reported by Baumann et al. Science 350, 417 (2015)]. The current and spin dynamics are modeled by a time-dependent Redfield master equation generalized to account for the periodic driven voltage. Our approach shows that the resonance effect is a consequence of the nonlinearity of the coupling between the magnetic moment and the spin-polarized current which generates a large second-harmonic amplitude that can be measured in the current signal.
\end{abstract}

\section{INTRODUCTION}

The interplay between the electronic motion and spin degrees of freedom is a key ingredient to design atomicsize magnetic devices. Recently, experimental advances have allowed a number of remarkable results in nonmagnetic control over single magnetic molecules and other artificially fabricated spin structures [1, 2]. This progress is significantly driven by future-technology demands, as single magnetic atoms have long been viewed as structural elements for high-density information storage and processing devices [3]. Recently, these structures were shown to preserve quantum coherence under certain circumstances 8, 9, which triggered a renewed interest in their use for quantum information processing [10].

Individual addressing of the atomic spin can be achieved only by non-magnetic means, using an external current applied locally at the atomic site [11 13. The degree of control over the atomic spin is ultimately determined by the nature of the coupling between the current and the magnetic moment. Therefore, it is of foremost importance to explore different coupling mechanisms. Reference [14] reports the current control of $\mathrm{TbPc}_{2}$ magnetic properties by applying controlled current pulses via a scanning tunneling microscope (STM) setup. The physical origin of this effect is a currentinduced molecular conformation. Recently, Baumann et al. 15. have reported the induction of a paramagnetic resonance of individual magnetic atoms on a surface by application of an alternating current. Several proposals have been put forward to understand this effect [15 17], which involves coupling the individual magnetic atom to mechanical (orbital) degrees of freedom that in turn couple to the alternating field. However, such coupling should be highly dependent on the local environment of the atom and thus these scenarios have difficulties explaining the ubiquity of the observations.

In this paper, we show that a current-induced spin

* a.shakirov@rqc.ru torque can effectively couple the spin of the magnetic atom to a locally applied alternating voltage. By modeling the experimental setup of Ref. [15], we argue that this mechanism can be responsible for the observed paramagnetic resonance and is compatible with the measured decoherence and decay times. The dissipative nature of the current-induced spin torque renders this effect distinct from mechanisms that induce a time dependence to the Hamiltonian of the local moment.

Baumann et al. [15] deposited a single magnetic atom (Fe or $\mathrm{Co}$ ) on a metallic surface, $\mathrm{Ag}(001)$, coated with a thin insulator, an atomically thick $\mathrm{MgO}$ layer. A sketch of the setup is given in Fig. 11. With a STM tip placed on top of the atom, an alternating voltage $(\sim 2-3 \mathrm{MHz})$ was applied between the tip and the metallic substrate, in addition to a direct voltage component. While swiping the frequency of the applied ac signal, a peak in the dc current response was observed marking a single magnetic excitation. The effect was shown to be present for a spinpolarized tip on $\mathrm{Fe}$ atoms and absent for Co atoms or for a spin-unpolarized tip. The width of the resonance measured in the current signal was related with $T_{2}$ and shown to be much smaller then the measured relaxation time $T_{1}$. Similar results were subsequently reported in Refs. [18] and [19].

Although we do not intend to reproduce the exact conditions of the experiment, we consider below a minimal model able to capture the main physical effects.

\section{MODEL \& METHOD}

A description of magnetic atoms and molecules in terms of an effective spin Hamiltonian emerges in the presence of large charge gap that effectively promotes the number of atomic electrons to a good quantum number. At low temperatures, in the regime of weak hybridization between the localized orbitals and the nearby itinerant electrons, tunneling arises by virtual excitations of the localized charge state giving rise to an effective exchange interaction between the localized spin and that of the electronic environment. This situation can be modeled 

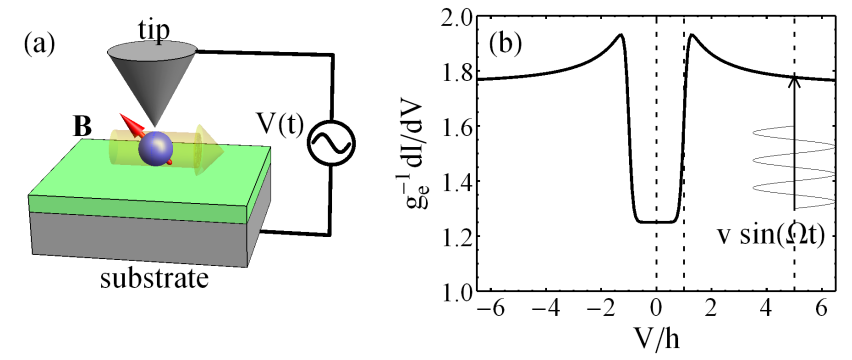

Figure 1. (a) The sketch of the setup that consists of the magnetic atom, the metallic substrate coated by the insulating layer, and the polarized tip. The external magnetic field $\boldsymbol{B}$ acts on the atom, and the periodically changing voltage $V(t)=V+v \sin (\Omega t)$ is applied across the tip. (b) The dc conductance spectrum of the setup. The sine curve indicates the range of values of the instant voltage.

by the Hamiltonian $H=H_{S}+H_{E}+H_{C}$, which includes the atomic subsystem $H_{S}$, the electronic degrees of freedom of the environment $H_{E}$, and the coupling term $H_{C}$. Here, the electronic environment consists of two metallic leads (substrate and tip) in thermal equilibrium with inverse temperature $\beta$ and chemical potentials $\mu_{s}=0$ and $\mu_{t}=-V$, where $V$ is the dc component of the applied voltage, and we set $e=1$ for the electron charge $-e$. The full voltage across the device

$$
V(t)=V+v \sin (\Omega t)
$$

also includes an alternating component with amplitude $v$ and frequency $\Omega$. We account for this component by adding a constant shift $-\Delta_{\nu} \sin (\Omega t)$, with $\Delta_{t}=v$ and $\Delta_{s}=0$, to the energies of the lead states, which renders the Hamiltonian of the tip time-dependent. The leads are also characterized by spin-polarized density of states (DOS) that account for the tip polarization and a bandwidth $W$, much larger than any energy scale of the system (for further details see Ref. [20]). The degree of the tip polarization is determined by the parameter $p$ that ranges from -1 to 1 . The atomic system consists of a single atom with a well-defined total spin $S=1 / 2$ in the presence of an external magnetic field $\boldsymbol{B}$,

$$
H_{S}=\boldsymbol{h} \cdot \boldsymbol{S}
$$

where $\boldsymbol{h}=g \mu_{B} \boldsymbol{B}$ is proportional to the atomic $g$-factor and the Bohr magneton $\mu_{B}$. The system-environment coupling Hamiltonian is given by the exchange interaction terms [21, 22]

$$
\begin{aligned}
H_{C} & =\sum_{a \nu \nu^{\prime}} \sqrt{J_{\nu}^{a} J_{\nu^{\prime}}^{a}} S_{a} \otimes s_{a}^{\nu \nu^{\prime}}, \\
s_{a}^{\nu \nu^{\prime}} & =\frac{1}{\sqrt{\mathcal{N}_{\nu} \mathcal{N}_{\nu^{\prime}}}} \sum_{k k^{\prime} s s^{\prime}} c_{\nu k s}^{\dagger} \frac{\sigma_{s s^{\prime}}^{a}}{2} c_{\nu^{\prime} k^{\prime} s^{\prime}},
\end{aligned}
$$

where $a=0, x, y, z$, the axis $z$ is aligned with the tip polarization, and $\sigma^{a}$ are the Pauli matrices (with $\sigma^{0}=S_{0}=I$ ). Therefore, terms with $a=0$ correspond to the elastic tunneling of electrons between the leads. The inelastic coupling is isotropic, i.e., $J_{\nu}^{a}=J_{\nu}$. In the following, we use dimensionless coupling parameters $\Gamma_{\nu}^{a}=\pi J_{\nu}^{a} / 2 W$, with $W$ the bandwidth of the reservoirs, and consider only the isotropic case $\Gamma_{\nu}^{a}=\Gamma_{\nu}$.

To capture spin-torque effects, we employ a masterequation description for the evolution of the reduced density matrix of the local moment that crucially includes the coherences. Therefore, we generalized the Redfield master equation approach, previously used to model coherent evolution and transport in engineered atomic spin devices [20, 23], to deal with the ac driving bias.

Following a standard procedure [24], a master equation $\partial_{t} \rho=\mathcal{L}_{t} \rho$ can be derived for the density matrix of the atomic system, where $\mathcal{L}_{t}$ is a superoperator of the Redfield type given by

$$
\mathcal{L}_{t} \rho=-i\left[H_{S}^{\prime}, \rho\right]-\sum_{a a^{\prime}}\left[S_{a}, \Lambda_{a a^{\prime}}(t) \rho-\rho \Lambda_{a a^{\prime}}^{\dagger}(t)\right] .
$$

Here $H_{S}^{\prime}=H_{S}+\frac{1}{2} p J_{t} S_{z}$ is the renormalized Hamiltonian of the system, and

$$
\begin{aligned}
\Lambda_{a a^{\prime}}(t)= & \sum_{\nu \nu^{\prime} \alpha \alpha^{\prime}} u_{a a^{\prime}}^{\nu \nu^{\prime}} \kappa_{\nu \nu^{\prime}}^{t}\left(\omega_{\alpha}-\omega_{\alpha^{\prime}}\right) \\
& \times|\alpha\rangle\left\langle\alpha\left|S_{a^{\prime}}\right| \alpha^{\prime}\right\rangle\left\langle\alpha^{\prime}\right|,
\end{aligned}
$$

where $u_{a a^{\prime}}^{\nu \nu^{\prime}}=\frac{\sqrt{\Gamma_{\nu}^{a} \Gamma_{\nu^{\prime}}^{a} \Gamma_{\nu}^{a^{\prime}} \Gamma_{\nu^{\prime}}^{a^{\prime}}}}{4 \pi} \operatorname{tr}\left[\left(1+p_{\nu} \sigma^{z}\right) \sigma^{a}\left(1+p_{\nu^{\prime}} \sigma^{z}\right) \sigma^{a^{\prime}}\right]$, and $|\alpha\rangle$ are eigenstates of $H_{S}^{\prime}$ with energies $\omega_{\alpha}$. The time dependence enters $\mathcal{L}_{t}$ via the quantity

$$
\begin{aligned}
\kappa_{\nu \nu^{\prime}}^{t}(\omega)= & \sum_{m=-\infty}^{\infty} i^{m} e^{i m \Omega t} e^{i \frac{\Delta_{\nu}-\Delta_{\nu^{\prime}}}{\Omega} \cos (\Omega t)} \\
& \times J_{m}\left(-\frac{\Delta_{\nu}-\Delta_{\nu^{\prime}}}{\Omega}\right) \kappa\left(\omega+m \Omega-\mu_{\nu}+\mu_{\nu^{\prime}}\right),
\end{aligned}
$$

where $J_{m}(x)$ are Bessel functions, that is the generalization of the one obtained in the time independent case [20]

$$
\kappa(\omega)=\frac{g(\beta \omega)+i f(\beta \omega)}{\beta}-\frac{i}{\pi} \omega \ln \frac{|\omega|}{c W},
$$

where $c$ is a constant of order $1, g(x)=x /\left(e^{x}-1\right)$, and $f(x)=\frac{1}{\pi} P \int d y[g(y)+y \Theta(-y)] /(x-y)$. The details of the derivation are given in Appendix $\mathrm{A}$ and are a generalization of the method of Ref. [20, obtained for a static voltage (i.e., $v=0)$, when $\kappa_{\nu \nu^{\prime}}^{t}(\omega)=\kappa\left(\omega-\mu_{\nu}+\mu_{\nu^{\prime}}\right)$ and the operators $\Lambda_{a a^{\prime}}(t)$ are time independent. For simplicity, the calculations below do not take into account the imaginary part of $\kappa(\omega)$. It is worth noting that this term may induce unphysical dynamics of the density matrix for large and moderate system-environment coupling, while it does not qualitatively change observables for weak coupling [20].

The average value of the current between the leads can be obtained introducing a counting field in the master 
equation (see Ref. [25] and Appendix A, or using a charge-specific formalism [20, 26, 27] adapted to the timedependent case, and is given by

$$
I(t)=-\operatorname{tr} \sum_{a a^{\prime}}\left[J_{a a^{\prime}}(t) \rho(t) S_{a}+S_{a} \rho(t) J_{a a^{\prime}}^{\dagger}(t)\right],
$$

where the operators $J_{a a^{\prime}}(t)$ are defined as

$$
\begin{aligned}
J_{a a^{\prime}}(t) & =\sum_{\nu \nu^{\prime} \alpha \alpha^{\prime}} u_{a a^{\prime}}^{\nu \nu^{\prime}} \kappa_{\nu \nu^{\prime}}^{t}\left(\omega_{\alpha}-\omega_{\alpha^{\prime}}\right) \\
& \times\left(\delta_{\nu t}-\delta_{\nu^{\prime} t}\right)|\alpha\rangle\left\langle\alpha\left|S_{a^{\prime}}\right| \alpha^{\prime}\right\rangle\left\langle\alpha^{\prime}\right|
\end{aligned}
$$

The expression Eq. (9) has the same form as the one obtained for the static case in Ref. [23], except for the explicit time dependence of the density matrix and the operators $J_{a a^{\prime}}(t)$ due to the driving. Note that the current obtained in this way assumes that the ac voltage has been turned on in the infinite past and that the system has already attained a periodic regime with the frequency of the drive. In practice, this means that the duration of the ac pulse is considered to be larger than the characteristic relaxation times. The average value of the current in Eq. (9) can be separated into three components [22, 28]: (i) the elastic component $I^{(1)}$ arising from the terms with $a=a^{\prime}=0$, (ii) the magnetoresistive component $I^{(2)}$ arising from the terms with $a=0$ and $a^{\prime} \neq 0$, or $a \neq 0$ and $a^{\prime}=0$, and (iii) the inelastic component $I^{(3)}$ arising from the terms with $a \neq 0$ and $a^{\prime} \neq 0$. We note that the elastic component has the trivial dependence on the voltage $I^{(1)}(t)=g_{e} V(t)$, satisfying the Ohm's law with $g_{e}=\Gamma_{s}^{0} \Gamma_{t}^{0} / \pi$.

\section{RESULTS}

We now apply the developed theory to study the electronic paramagnetic resonance in magnetic atoms. We first calculate the ac spectra of the current to demonstrate the appearance of the resonance peaks observed in Ref. [15]; furthermore, we investigate how the spin dynamics behaves in the vicinity of the resonance.

For clarity, we split the average value of the current as

$$
I(t)=I+i(t)
$$

where $I$ is the stationary current at constant voltage $V$, and $i(t)$ is the differential response to the ac component. Since the applied voltage Eq. (1) changes periodically, $i(t)$ admits the Fourier series decomposition:

$$
i(t)=i^{(0)}+\sum_{m=1}^{\infty} i^{(m)} \sin \left(m \Omega t+\phi_{m}\right)
$$

Following Baumann et al. [15], we first study $i^{(0)}$, whose dependence on the driving frequency for different values of the dc voltage $V$ and the driving amplitudes $v$ is shown in Fig. 2, When the spin polarization of the current is

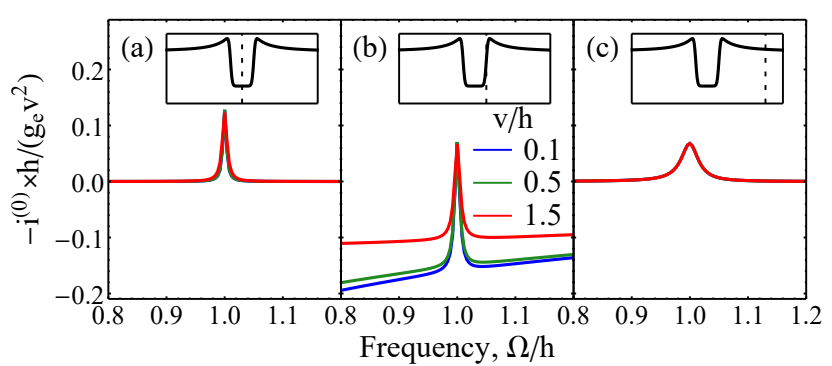

Figure 2. Dependence of $i^{(0)}$ term in the ac component of the current Eq. 12 on the driving frequency for different values of the dc voltage and the driving amplitude. We use $h=0.1$ $\mathrm{meV}, T=0.05 h, \Gamma_{\nu}^{a}=0.1$, and (a) $V=0$, (b) $V=h$, (c) $V=5 h$. Since $i^{(0)}$ scales as $v^{2}$, we normalize it appropriately. The insets show the spectrum of the dc current and the values of $V$ around which the voltage is driven.

perpendicular to the magnetic field applied to the atomic spin, we see that in all cases there is a pronounced peak at the resonant driving frequency $\Omega=h$. As in the experiment, such peak is not observed if the current polarization is collinear with the magnetic field $\boldsymbol{h}$. Note that $i^{(0)}$ in Fig. 2 is normalized by $v^{2}$, therefore, the collapse of these curves near the resonance frequency, for different driving amplitudes $v$ and different values of $V$, indicates that the non-linear processes generating the $i^{(0)}$ response are predominantly of second order in $v$. Away from the resonance the $i^{(0)}$ response drops sharply for $V=0$ and $5 h$. However, for $V=h$ one observes a non-zero $i^{(0)}$ response even off-resonance, this arises since the driving is done around the dc voltage that corresponds to a highly non-linear part of the spectrum as can be seen in the inset of Fig. 2(b).

We now extend our analysis to the full dynamics of the current within a driving period for the case (c) of Fig. 2 that best models the conditions reported in Ref. [15]. We study current response for driving frequencies $\Omega \ll h$ (adiabatic case) and $\Omega \gg h$ (fast case) and compare it with the resonant case, $\Omega=h$. For both adiabatic and fast driving we find that

$$
i(t) \simeq i_{0}(t)=\frac{d I}{d V}(V) \times v \sin \Omega t,
$$

i.e., the ac response is purely ohmic. The Lissajous curves depicting the nonohmic part of the response $i(t)-i_{0}(t)$ versus $v(t)=v \sin \Omega t$ are shown in Fig. 3(a) for different amplitudes and frequencies of the driving.

When the driving is adiabatic, i.e., $\Omega \ll h$, the instantaneous current $i(t)$ is solely determined by the instantaneous voltage at time $t$, in which case the Lissajous curve shows no hysteresis and can be determined from the dc curve. On the other hand, when the driving frequency is large, i.e., $\Omega \gg h$, the internal state of the system has no time to adapt (see below). The magnetic moment thus experiences vanishing time-averaged torque. The resulting conductivity, that is determined by the state of the magnetic moment, also does not depend on the time 

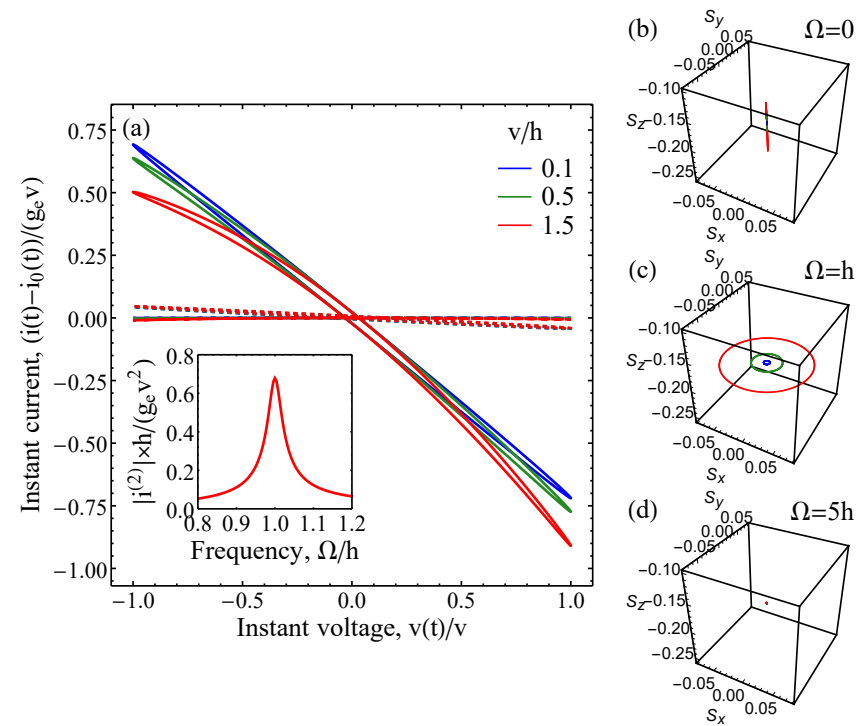

Figure 3. (a) Lissajous curves for $i(t)-i_{0}(t)$ versus $v(t)=$ $v \sin \Omega t$ and (b)-(d) over-period trajectories of the average atomic spin at different amplitudes and frequencies of the driving. Dashed, solid, and dotted curves correspond to adiabatic $(\Omega=0)$, resonant $(\Omega=h)$, and fast $(\Omega=5 h)$ driving cases. We use $h=0.1 \mathrm{meV}, T=0.05 h, \Gamma_{\nu}^{a}=0.1$, and $V=5 h$. The values of $v(t)$ and $i(t)-i_{0}(t)$ are normalized to $v$ and $g_{e} v$, respectively. The inset shows the amplitude of the second harmonic of the current $i^{(2)}$, normalized by $v^{2}$, as the function of the driving frequency for the same $h, T, \Gamma_{\nu}^{a}$, and $V$. Since $i^{(2)}$ scales as $v^{2}$, curves for different amplitudes of the driving $v$ are indistinguishable.

resulting in purely Ohmic response $i(t) \propto v(t)$. At resonance $\Omega=h$, the $i-v$ characteristic exhibits a hysteresis loop indicated by the non-vanishing area inside the Lissajous curve. This shows that the non-linear processes responsible for the generation of $i^{(0)}$ also induce higher harmonics whose amplitudes $i^{(m)}$ increase at resonance. The amplitude of the second harmonic $m=2$, shown in the inset of Fig. 3(a), is comparable to $i^{(0)}$ and also scales as $v^{2}$.

To better understand the phenomena reported above, we investigate the dynamics of the magnetic moment for three different regimes considered above. Figure 3 shows the orbit followed by the Bloch vector $s=$ $\left\{\left\langle S_{x}\right\rangle,\left\langle S_{y}\right\rangle,\left\langle S_{z}\right\rangle\right\}$ over a period of the drive. For adiabatic driving, $\Omega \ll h$, shown in Fig. 3(b), the spin has time to adapt to the applied voltage and its trajectory can be obtained by the static master equation. The magnetization points in the $z$ direction and oscillates around the static (i.e., $v=0$ ) value with an amplitude that is proportional to $v$. In the regime of fast driving, $\Omega \gg h$, shown in Fig. 3(d), the magnetization remains static and independent of $v$, acquiring the value obtained in the static case for $v=0$. This can be simply explained by the fact that, for the time scales experienced by the spin dynamics, the alternating voltage averages out to zero. The resonant case, $\Omega=h$, is shown in Fig. 3(c).
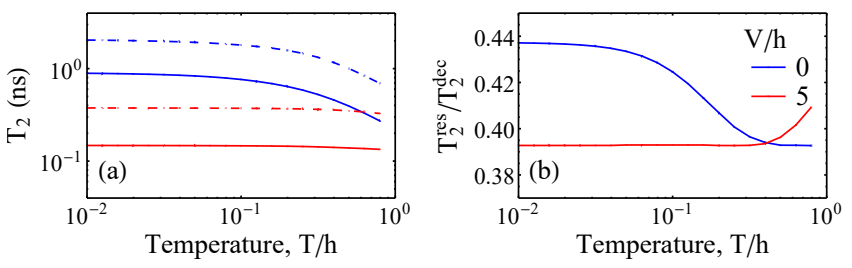

Figure 4. The dependence on the temperature of (a) the decoherence times $T_{2}^{\text {res }}$ (solid curves) and $T_{2}^{\text {dec }}$ (dashed curves) estimated from the width of the resonance and from the decay time of the coherence between the spin states, correspondingly, (b) their ratio $T_{2}^{\text {res }} / T_{2}^{\text {dec }}$. The timescales $T_{2}^{\text {res }}$ are obtained from fitting $i^{(0)}$ resonance curves for $h=0.1 \mathrm{meV}$, $v=0.1 h, \Gamma_{\nu}^{a}=0.1$, and different values of $V$.

The trajectories form circular orbits almost parallel to the $x y$ plane, centered at the static value, and with radii proportional to $v$. This shows that, at resonance, the perpendicular polarized current exerts a spin-transfer torque that is able to excite the magnetic moment of the atom. This process requires quantum coherence, as it involves the elements of the reduced density matrix of the magnetic moment that are off-diagonal with respect to the Hamiltonian.

Finally, we address another point raised by Ref. 15 concerning the quantum coherence of a spin state. In Ref. [15], the measurements of $i^{(0)}$ were used to indirectly evaluate the decoherence time $T_{2}$ by determining the width of the resonance. Such timescale can now be compared with the standard interpretation of $T_{2}$ as the decay time of the coherences of the spin state [29]. The definition of $T_{2}$ employed in the following has been established in Ref. [23], where some of the subtleties of defining a decoherence timescale in the presence of a spin-polarized environment were addressed. This quantity, dubbed $T_{2}^{\text {dec }}$ in the following, is determined by the fastest decay rate of information in a system perturbed away from the nonequilibrium steady state that is established in the presence of a static bias $V$.

The analog of $T_{2}$ as measured in Refs. 15, 18, that we denote $T_{2}^{\text {res }}$, is obtained from the width of the resonance curves in Figs. 2(a) and 2(c), assuming that near the resonance $i^{(0)}(\Omega) \propto e^{-b(\Omega-h)^{2}}$. The half-height width is then computed as $T_{2}^{\text {res }}=\frac{1}{2} \sqrt{\frac{b}{\ln 2}}$. Figure 4 depicts $T_{2}^{\text {res }}$, $T_{2}^{\text {dec }}$, and their ratio as a function of the temperature for two values of $V$. The fact that this ratio is constant at low temperature, and depends mildly on the $V$, shows that $T_{2}^{\text {res }}$ can indeed be used to estimate $T_{2}^{\text {dec }}$. However, both the variation observed at high temperatures and the dependence on $V$ can yield a difference of the order of $10 \%$ in the ratio $T_{2}^{\text {res }} / T_{2}^{\text {dec }}$. This suggests some caution to the assign a direct physical meaning to $T_{2}^{\text {res }}$.

The assumption of a spin- $1 / 2$ atom directly applies to Ref. [18. Nonetheless, our theoretical treatment and the effects it predicts generalize to higher magnetic moments, engineered spin structures, and magnetic molecules, pro- 
viding the jump operators in Eq. A5 directly couple the resonant energy states. To avoid this limitation, a higher order expansion in the system-bath coupling has to be considered.

\section{CONCLUSION}

In this paper, we prove that spin-transfer torque may induce an electronic paramagnetic resonance in single atomic spins. This mechanism does not appeal to any mechanical or orbital degrees of freedom and only involves the dissipative interaction of the polarized current with the atomic spin. It is therefore independent of the environment of the atom which may explain the ubiquity of the effect reported in Ref. [15]. The current and spin dynamics induced by an ac voltage drive can be well captured by a time-dependent master equation that generalizes a previous framework 20 based on a Redfield-like set of approximations. Our approach shows that the quantum coherence of the atomic spin is crucial to capture paramagnetic resonance effects resulting from the generation of second harmonics of the driving signal. This nonlinear process is enhanced near the resonance condition and depends on the square of the driving amplitude, which is compatible with Ref. [19]. The effect is based on a current-induced spin-torque and does not assume any effective time dependence of the local moment Hamiltonian. Moreover, the resonance is not observed for an unpolarized current which generates no spin-transfer torque.

We showed that the resonance width can reliably be used to estimate the decoherence timescale $T_{2}$ once mild temperature and voltage dependencies are accounted for.

\section{ACKNOWLEDGMENTS}

P.R. acknowledges support by FCT through the Investigador FCT Contract No. IF/00347/2014 and Grant No. UID/CTM $/ 04540 / 2013$. We thankfully acknowledge the computer resources, technical expertise and assistance provided by CENTRA/IST. Computations were performed at the cluster Baltasar-Sete-Sóis and supported by the H2020 ERC Consolidator Grant, "Matter and strong field gravity: New frontiers in Einstein's theory" grant agreement No. MaGRaTh-646597. The contribution of A.S. was funded by RFBR Grant No. 1642-01057.

\section{Appendix A: Redfield equation}

In this Appendix, we derive the Redfield equation that was used in the paper to calculate dynamics of the atom driven by the current. The driving is included into the Hamiltonian of the environment as a time-dependent shift to the energies of the electronic states

$$
H_{E}(t)=\sum_{\nu k s}\left[\varepsilon_{\nu k s}-\delta_{\nu}(t)\right] c_{\nu k s}^{\dagger} c_{\nu k s}
$$

where $\nu=s, t$ labels the leads, indices $k$ and $s$ enumerate momentum and spin of the electrons, and $\Delta_{\nu}(t)=$ $\Delta_{\nu} \sin (\Omega t)$. We start by representing the coupling Hamiltonian, see Eq. (3) of the paper, as $H_{C}=\sum_{a} S_{a} \otimes$ $E_{a}$ with

$$
E_{a}=\sum_{\nu \nu^{\prime}} \sqrt{\frac{J_{\nu}^{a} J_{\nu^{\prime}}^{a}}{\mathcal{N}_{\nu} \mathcal{N}_{\nu^{\prime}}}} \sum_{k k^{\prime} s s^{\prime}} c_{\nu k s}^{\dagger} \frac{\sigma_{s s^{\prime}}^{a}}{2} c_{\nu^{\prime} k^{\prime} s^{\prime}}
$$

which allows us to write the Redfield equation in the well-known form

$$
\begin{aligned}
\partial_{t} \rho & =-i\left[H_{S}+\Delta H_{S}, \rho\right] \\
& +\sum_{a a^{\prime}}\left(\left[\Lambda_{a a^{\prime}}(t) \rho, S_{a}\right]+\text { H.c. }\right) .
\end{aligned}
$$

Here the Hamiltonian shift is given by

$$
\Delta H_{S}=\sum_{a}\left\langle E_{a}\right\rangle S_{a}
$$

and the operators

$$
\Lambda_{a a^{\prime}}(t)=\int_{0}^{\infty} d t^{\prime} e^{-i H_{S} t^{\prime}} S_{a^{\prime}} e^{i H_{S} t^{\prime}} C_{a a^{\prime}}\left(t, t^{\prime}\right)
$$

are expressed through the correlation functions of the environment

$$
\begin{aligned}
C_{a a^{\prime}}\left(t, t^{\prime}\right) & =\operatorname{tr}_{E}\left[\left(E_{a}-\left\langle E_{a}\right\rangle\right) e^{-i \int_{t-t^{\prime}}^{t} H_{E}(\tau) d \tau}\right. \\
& \left.\times\left(E_{a^{\prime}}-\left\langle E_{a^{\prime}}\right\rangle\right) e^{i \int_{t-t^{\prime}}^{t} H_{E}(\tau) d \tau} \rho_{E}\right] .
\end{aligned}
$$

Evaluating the Hamiltonian shift results in

$$
\Delta H_{S}=\sum_{\nu a} \frac{J_{\nu}^{a}}{\mathcal{N}_{\nu}} \sum_{k s} \frac{\sigma_{s s}^{a}}{2} n_{F}\left(\varepsilon_{\nu k s}-\mu_{\nu}\right) S_{a} .
$$

We employ spin-polarized DOS of the leads,

$$
\begin{aligned}
\varrho_{\nu s}(\varepsilon) & =\frac{1}{\mathcal{N}_{\nu}} \sum_{k} \delta\left(\varepsilon-\varepsilon_{\nu k s}\right) \\
& =\frac{1}{2 W}\left(1+p_{\nu} s\right) \Theta\left(\left|\varepsilon-\mu_{\nu}\right|-W\right),
\end{aligned}
$$

to replace sums over momenta by integrals over energy in this expression and obtain

$$
\begin{aligned}
\Delta H_{S} & =\frac{1}{2} \sum_{\nu} \int d \varepsilon\left[\left(J_{\nu}^{0}+J_{\nu} S_{z}\right) \varrho_{\nu \uparrow}(\varepsilon)\right. \\
& \left.+\left(J_{\nu}^{0}-J_{\nu} S_{z}\right) \varrho_{\nu \downarrow}(\varepsilon)\right] n_{F}\left(\varepsilon-\mu_{\nu}\right) .
\end{aligned}
$$

In the large bandwidth limit, with constant terms discarded, one gets

$$
\Delta H_{S}=\frac{1}{2} \sum_{\nu} J_{\nu} p_{\nu} S_{z}
$$


or $\Delta H_{S}=\frac{1}{2} J_{t} p S_{z}$ for the case when only tip is polarized. Because of driving, the correlation functions depend on both time arguments rather than their difference. Substituting Eq. A2 into Eq. A6 results in

$$
\begin{aligned}
C_{a a^{\prime}}\left(t, t^{\prime}\right) & =\sum_{\nu \nu^{\prime}} \frac{\sqrt{J_{\nu}^{a} J_{\nu^{\prime}}^{a} J_{\nu}^{a^{\prime}} J_{\nu^{\prime}}^{a^{\prime}}}}{\mathcal{N}_{\nu} \mathcal{N}_{\nu^{\prime}}} \sum_{k k^{\prime} s s^{\prime}} \frac{\sigma_{s s^{\prime}}^{a} \sigma_{s^{\prime} s}^{a^{\prime}}}{4} \\
& \times\left\langle c_{\nu k s}^{\dagger} c_{\nu^{\prime} k^{\prime} s^{\prime}} e^{-i \int_{t-t^{\prime}}^{t} H_{E}(\tau) d \tau}\right. \\
& \left.\times c_{\nu^{\prime} k^{\prime} s^{\prime}}^{\dagger} c_{\nu k s} e^{i \int_{t-t^{\prime}}^{t} H_{E}(\tau) d \tau}\right\rangle .
\end{aligned}
$$

Introducing DOS of the leads, we replace sums over momenta by integrals over energy in this expression and obtain

$$
\begin{aligned}
C_{a a^{\prime}}\left(t, t^{\prime}\right) & =\sum_{\nu \nu^{\prime}} \sqrt{J_{\nu}^{a} J_{\nu^{\prime}}^{a} J_{\nu}^{a^{\prime}} J_{\nu^{\prime}}^{a^{\prime}}} \sum_{s s^{\prime}} \frac{\sigma_{s s^{\prime}}^{a} \sigma_{s^{\prime} s}^{a^{\prime}}}{4} \\
& \times \iint d \varepsilon d \varepsilon^{\prime} \varrho_{\nu s}(\varepsilon) \varrho_{\nu^{\prime} s^{\prime}}\left(\varepsilon^{\prime}\right) \\
& \times e^{i \int_{t-t^{\prime}}^{t}\left[\varepsilon-v_{\nu}(\tau)\right] d \tau} e^{-i \int_{t-t^{\prime}}^{t}\left[\varepsilon^{\prime}-v_{\nu^{\prime}}(\tau)\right] d \tau} \\
& \times n_{F}\left(\varepsilon-\mu_{\nu}\right)\left[1-n_{F}\left(\varepsilon-\mu_{\nu^{\prime}}\right)\right] .
\end{aligned}
$$

We then employ rectangular DOS, introduce dimensionless coupling parameters $\Gamma_{\nu}^{a}=\pi J_{\nu}^{a} /(2 W)$, and use the relation $1-n_{F}(\varepsilon)=n_{F}(-\varepsilon)$ to rewrite the last expression as

$$
\begin{aligned}
C_{a a^{\prime}}\left(t, t^{\prime}\right) & =\sum_{\nu \nu^{\prime}} \frac{1}{4 \pi} \sqrt{\Gamma_{\nu}^{a} \Gamma_{\nu^{\prime}}^{a} \Gamma_{\nu}^{a^{\prime}} \Gamma_{\nu^{\prime}}^{a^{\prime}}} \\
& \times \operatorname{tr}\left[\left(1+p_{\nu} \sigma^{z}\right) \sigma^{a}\left(1+p_{\nu^{\prime}} \sigma^{z}\right) \sigma^{a^{\prime}}\right] \\
& \times e^{i\left(\mu_{\nu}-\mu_{\nu^{\prime}}\right) t^{\prime}-i \int_{t-t^{\prime}}^{t}\left[v_{\nu}(\tau)-v_{\nu^{\prime}}(\tau)\right] d \tau} \\
& \times \frac{1}{\pi}\left[\int_{-W}^{W} d \varepsilon e^{i \varepsilon t^{\prime}} n_{F}(\varepsilon)\right]^{2}
\end{aligned}
$$

Let us introduce the definition

$$
\begin{aligned}
u_{a a^{\prime}}^{\nu \nu^{\prime}} & =\frac{1}{4 \pi} \sqrt{\Gamma_{\nu}^{a} \Gamma_{\nu^{\prime}}^{a} \Gamma_{\nu}^{a^{\prime}} \Gamma_{\nu^{\prime}}^{a^{\prime}}} \\
& \times \operatorname{tr}\left[\left(1+p_{\nu} \sigma^{z}\right) \sigma^{a}\left(1+p_{\nu^{\prime}} \sigma^{z}\right) \sigma^{a^{\prime}}\right]
\end{aligned}
$$

and substitute Eq. A13 into Eq. A5 using the spectral decomposition $I=\sum_{\alpha}|\alpha\rangle\langle\alpha|$. We get

$$
\begin{aligned}
\Lambda_{a a^{\prime}}(t) & =\sum_{\alpha \alpha^{\prime}}|\alpha\rangle\left\langle\alpha\left|S_{a}\right| \alpha^{\prime}\right\rangle\left\langle\alpha^{\prime}\right| \\
& \times \sum_{\nu \nu^{\prime}} u_{a a^{\prime}}^{\nu \nu^{\prime}} \int_{0}^{\infty} d t^{\prime} e^{-i\left(\omega_{\alpha}-\omega_{\alpha^{\prime}}-\mu_{\nu}+\mu_{\nu^{\prime}}\right) t^{\prime}} \\
& \times e^{-i \int_{t-t^{\prime}}^{t}\left[v_{\nu}(\tau)-v_{\nu^{\prime}}(\tau)\right] d \tau} \\
& \times \frac{1}{\pi}\left[\int_{-W}^{W} d \varepsilon e^{i \varepsilon t^{\prime}} n_{F}(\varepsilon)\right]^{2}
\end{aligned}
$$

which coincides with Eq. A5 of the paper, where

$$
\begin{aligned}
\kappa_{\nu \nu^{\prime}}^{t}(\omega) & =\int_{0}^{\infty} d t^{\prime} e^{-i\left(\omega-\mu_{\nu}+\mu_{\nu^{\prime}}\right) t^{\prime}} \\
& \times e^{-i \int_{t-t^{\prime}}^{t}\left[v_{\nu}(\tau)-v_{\nu^{\prime}}(\tau)\right] d \tau} \\
& \times \frac{1}{\pi}\left[\int_{-W}^{W} d \varepsilon e^{i \varepsilon t^{\prime}} n_{F}(\varepsilon)\right]^{2} .
\end{aligned}
$$

For sinusoidal periodic driving $v_{\nu}(t)=v_{\nu} \sin (\Omega t)$, we may decompose

$$
\begin{aligned}
& e^{-i \int_{t-t^{\prime}}^{t}\left[v_{\nu}(\tau)-v_{\nu^{\prime}}(\tau)\right] d \tau} \\
& =e^{i \frac{v_{\nu}-v_{\nu^{\prime}}}{\Omega}\left[\cos (\Omega t)-\cos \left(\Omega\left(t-t^{\prime}\right)\right)\right]} \\
& =e^{i \frac{v_{\nu}-v_{\nu^{\prime}}}{\Omega} \cos (\Omega t)} \sum_{m=-\infty}^{+\infty} i^{m} J_{m}\left(-\frac{v_{\nu}-v_{\nu^{\prime}}}{\Omega}\right) e^{i m \Omega\left(t-t^{\prime}\right),}
\end{aligned}
$$

where we used the identity $e^{i z \cos \varphi}=$ $\sum_{m=-\infty}^{+\infty} i^{m} J_{m}(z) e^{i m \varphi}$. Substituting this into Eq. (A16) gives Eq. (7) of the paper with

$$
\kappa(\omega)=\frac{1}{\pi} \int_{0}^{\infty} d t e^{-i \omega t}\left[\int_{-W}^{W} d \varepsilon e^{i \varepsilon t} n_{F}(\varepsilon)\right]^{2} .
$$

The evaluation of this integral in the large bandwidth limit $W \rightarrow \infty$ results in

$$
\begin{aligned}
\kappa(\omega) & =-\frac{i}{\pi}\left(b W+\omega \ln \frac{|\omega|}{c W}\right) \\
& +\frac{g(\beta \omega)+i f(\beta \omega)}{\beta}+O\left(\frac{|\omega|}{W}\right),
\end{aligned}
$$

where $b=2 \ln 2$ and $c=e / 2$. We note that DOS with different from rectangular shapes give $\kappa(\omega)$ of the same form but with other values of $b$ and $c$, e.g., for $\varrho_{\nu s}(\varepsilon)=$ $\frac{1}{2 W}\left(1+p_{\nu} s\right) \exp \left(-\left|\varepsilon-\mu_{\nu}\right| / W\right)$ one gets $b=1$ and $c=$ $e^{-\gamma}$. The term proportional to the bandwidth gives no contribution to the equation and we thus exclude it from $\kappa(\omega)$, as well as $O(|\omega| / W)$ term, and arrive at Eq. (8) of the paper. 
[1] Editorial, Nat. Nanotechnol. 10, 185 (2015)

[2] J. L. Zhang, J. Q. Zhong, J. D. Lin, W. P. Hu, K. Wu, G. Q. Xu, A. T. S. Wee, and W. Chen, Chem. Soc. Rev. 44, $2998(2015)$

|3| F. Troiani, A. Ghirri, M. Affronte, S. Carretta, P. Santini, G. Amoretti, S. Piligkos, G. Timco, and R. E. P. Winpenny, Phys. Rev. Lett. 94, 207208 (2005)

[4] A. Imre, Science 311, 205 (2006)

[5] L. Bogani and W. Wernsdorfer, Nat. Mater. 7, 179 (2008)

[6] A. A. Khajetoorians, J. Wiebe, B. Chilian, and R. Wiesendanger, Science 332, 1062 (2011).

[7] F. D. Natterer, K. Yang, W. Paul, P. Willke, T. Choi, T. Greber, and A. J. Heinrich, Nature 543, 226 (2017).

[8] B. W. Heinrich, L. Braun, J. I. Pascual, and K. J. Franke, Nat. Phys. 9, 765 (2013).

[9] D. A. Lidar, in Quantum Information and Computation for Chemistry: Advances in Chemical Physics, Vol. 154 (John Wiley \& Sons, Inc., Hoboken, 2014) pp. 295-354.

[10] A. Ghirri, A. Candini, and M. Affronte, Magnetochemistry 3, 12 (2017)

[11] F. Meier, L. Zhou, J. Wiebe, and R. Wiesendanger, Science 320, 82 (2008)

[12] R. Wiesendanger, Rev. Mod. Phys. 81, 1495 (2009).

[13] S. Loth, K. von Bergmann, M. Ternes, A. F. Otte, C. P. Lutz, and A. J. Heinrich, Nat. Phys. 6, 340 (2010).

[14] T. Komeda, H. Isshiki, J. Liu, Y. F. Zhang, N. Lorente, K. Katoh, B. K. Breedlove, and M. Yamashita, Nat. Commun. 2, 217 (2011).

[15] S. Baumann, W. Paul, T. Choi, C. P. Lutz, A. Ardavan, and A. J. Heinrich, Science 350, 417 (2015).

[16] P. Berggren and J. Fransson, Sci. Rep. 6, 25584 (2016)
[17] J. L. Lado, A. Ferrón, and J. Fernández-Rossier, Phys. Rev. B 96, 205420 (2017)

[18] K. Yang, Y. Bae, W. Paul, F. D. Natterer, P. Willke, J. L. Lado, A. Ferrón, T. Choi, J. Fernández-Rossier, A. J. Heinrich, and C. P. Lutz, Phys. Rev. Lett. 119, 227206 (2017)

[19] P. Willke, W. Paul, F. D. Natterer, K. Yang, Y. Bae, T. Choi, J. F. Rossier, A. J. Heinrich, and C. P. Lutz, Sci. Adv. 4, eaaq1543 (2018)

[20] A. M. Shakirov, Y. E. Shchadilova, A. N. Rubtsov, and P. Ribeiro, Phys. Rev. B 94, 224425 (2016)

[21] G. H. Kim and T. S. Kim, Phys. Rev. Lett. 92, 137203 (2004)

[22] J. Fernández-Rossier, Phys. Rev. Lett. 102, 256802 (2009)

23] A. M. Shakirov, A. N. Rubtsov, A. I. Lichtenstein, and P. Ribeiro, Phys. Rev. B 96, 094410 (2017)

[24] H.-P. Breuer and F. Petruccione, The Theory of Open Quantum Systems (Oxford University Press, Oxford, 2002).

[25] Y. V. Nazarov, ed., Quantum Noise in Mesoscopic Physics, Vol. 97 (Springer, Netherlands, 2003).

[26] J. Rammer, A. L. Shelankov, and J. Wabnig, Phys. Rev. B 70, 115327 (2004)

[27] C. Flindt, T. Novotný, and A.-P. Jauho, Europhys. Lett. 69, 475 (2005).

[28] F. Delgado and J. Fernández-Rossier, Phys. Rev. B 82, 134414 (2010)

[29] F. Delgado and J. Fernández-Rossier, Progr. Surf. Sci. 92, 40 (2017). 